\title{
Nucleophile Addition of Reduced Glutathione on 2-Methyl-2-Nitroso Compound: A Combined Electron Paramagnetic Resonance Spectroscopy and Electrospray Tandem Mass Spectrometry Study
}

\author{
Mathilde Triquigneaux, Béatrice Tuccio, Robert Lauricella, \\ and Laurence Charles \\ Universités Aix-Marseille I, II \& III - CNRS, UMR 6264: Laboratoire Chimie Provence, Spectrométries \\ Appliquées à la Chimie Structurale, Marseille, France
}

\begin{abstract}
Mass spectrometry (MS) was used in conjunction with electron paramagnetic resonance (EPR) to characterize products arising from reactions between reduced glutathione (GSH) and 2-methyl 2-nitroso propane (MNP) in an oxidative medium, to evaluate the reactivity of this tripeptide as a nucleophile toward a nitroso compound. Depending on the experimental conditions, different radical species could be detected by EPR, which allowed some structural assumptions. These samples were then submitted to electrospray ionization, in both positive and negative ion modes, for structural elucidation in tandem mass spectrometry. Although the primary nitroxide products could not be detected in MS, structurally related compounds such as hydroxylamine and O-methyl hydroxylamine could be fully characterized. In the absence of light, a $S$-adduct was formed via a Forrester-Hepburn reaction, that is, a nucleophile addition of MNP onto the thiol function in reduced glutathione to yield a hydroxylamine intermediate, further oxidized into nitroxide. In contrast, irradiating the reaction medium with visible light could allow an inverted spin trapping reaction to take place, involving the oxidation of both MNP and GSH before the nucleophilic addition of the sulfenic acid function onto the nitrogen of MNP, yielding a so-called $\mathrm{O}$-adduct. It was also found that dilution of the reaction medium with methanol for the purpose of electrospray ionization could allow nitroxides to be indirectly observed either as hydroxylamine or O-methyl hydroxylamine species. (J Am Soc Mass Spectrom 2009, 20, 2013-2020) (C 2009 American Society for Mass Spectrometry
\end{abstract}

$\mathrm{R}$ educed glutathione (GSH), a natural tripeptide containing cysteine, glutamic acid, and glycine, presents a multitude of essential physiologic properties such as redox-buffering of the cell environment, detoxification of xenobiotic, and antioxidant regulator, which would depend on the reactivity of the cysteinyl thiol group [1]. GSH is known as a substrate in both conjugation and reduction reactions catalyzed by glutathione S-transferase enzymes [2,3]. It can also be involved in nonenzymatic conjugation reactions, yielding potentially toxic by-products [4,5]. In both types of conjugation, reduced glutathione can add to a double bond of aromatic compounds [6], to an epoxide group, to an isocyanate carbon [7], or substitute a halogen atom in alkyl- or aryl-halides [8]. Methods for the detection and characterization of metabolites arising from reac-

Address reprint requests to Dr. L. Charles, Universités Aix-Marseille I, II \& III - CNRS, UMR 6264: Laboratoire Chimie Provence, Spectrométries Appliquées à la Chimie Structurale, Campus Saint-Jérôme, Case 511, 13397 Marseille Cedex 20, France. E-mail: laurence.charles@univ-provence.fr tions involving GSH were thus developed, in particular using a mass spectrometric approach [9].

In tandem mass spectrometry (MS/MS), while the nature of the species conjugated to glutathione was shown to dramatically influence the gas-phase ion fragmentation pathways, some similarities were also demonstrated [9-12]. In particular, in the positive ion mode, a main dissociation reaction consists of the elimination of a $129 \mathrm{Da}$ neutral corresponding to a pyroglutamic acid molecule. As a result, detection of glutathione conjugates is often performed using a 129 Da neutral loss MS/MS experiments [13-17]. Nevertheless, different classes of glutathione conjugates, such as aliphatic thioether adducts ionized in the positive ion mode, were shown to behave differently upon collisioninduced dissociation (CID) [18, 19]. An alternative approach was reported using the negative ion mode with a characteristic MS/MS reaction, yielding the deprotonated $\gamma$-glutamyl-dehydroalanyl-glycine at $\mathrm{m} / \mathrm{z} 272$, shown to occur for any glutathione conjugates [19]. The usefulness of this approach was demonstrated in a variety of GSH 
adducts obtained from the reaction between reactive metabolites and this tripeptide [19].

All glutathione conjugates reported so far in the literature were synthesized either by endogenous or exogenous non radical processes. However, glutathione presents other activities, in particular as a powerful cellular antioxidant [20]. The thiyl radical generated by $\mathrm{H}^{\bullet}$ exchange with reactive oxygen species (ROS) free radicals could further be trapped with compounds such as nitrones [21-23] or nitroso-compounds [23-25], and characterized by electron paramagnetic resonance (EPR) detection of the so-produced nitroxide. Moreover, the GSH nucleophilic sulfur is also known to interact with the electrophilic nitrogen of nitrosoarenes to yield hemimercaptals [26-29]. However, these compounds are unstable and readily produce sulfinic acid, amine, $N$-hydroxyamine or arylamine, depending on both the reaction conditions ( $\mathrm{pH}$, buffer concentration) and the nature of other substituents on the aromatic ring.

We propose here to study the reactivity of reduced glutathione as a nucleophile toward an alkyl nitroso compound, namely 2-methyl 2-nitrosopropane (MNP), and to scrutinize the mechanism of the observed reactions by characterizing the products using EPR in conjunction with mass spectrometry. Such an analytical approach was previously demonstrated to be a very efficient tool to identify, without preliminary chromatographic separation, nitroxides or dismutation compounds obtained after spin trapping reactions using nitrones $[30,31]$.

\section{Experimental}

\section{Chemicals}

Reduced glutathione ( $>98 \%$ ) and ammonium acetate (98\%) were purchased from Sigma-Aldrich (Saint Louis, MO, USA). 2-Methyl-2-nitrosopropane (MNP) dimer (98\%) was purchased from Acros, Thermo Fisher Scientific, (Waltham, MA, USA) and $\mathrm{H}_{2} \mathrm{O}_{2}(30 \%)$ was from VWR (Fontenay sous Bois, France). Methanol (SDS; Peypin, France) and benzene (Sigma-Aldrich) were of the highest grade of purity commercially available and used as received. Deuterated methanol $\left(\mathrm{CD}_{3} \mathrm{OD}\right)$ was from Eurisotop (Saint-Aubin, France). Poly(ethylene glycol) with $M_{\mathrm{n}} 400$ g.mol ${ }^{-1}$ (Sigma-Aldrich) was used as an internal standard for accurate mass measurements.

\section{Electron Paramagnetic Resonance}

EPR assays were carried out at room-temperature in capillary tubes by using a computer-controlled EMX spectrometer (Bruker BioSpin; Silberstreifen, Germany) operating at X-band with $100 \mathrm{kHz}$ modulation frequency. The instrument settings were as follows: nonsaturating microwave power, $20 \mathrm{~mW}$; modulation amplitude, $2 \mathrm{G}$; receiver gain, $1 \times 10^{6}$; time constant, 1.28 ms; scan time, 120 s; scan width, 100 G; 1 scan. Com- puter simulation of the EPR spectra were achieved using the program elaborated by Duling [32].

\section{Mass Spectrometry}

High-resolution MS and MS/MS experiments were performed with a QStar Elite mass spectrometer (Applied Biosystems SCIEX, Concord, ON, Canada) equipped with an electrospray ionization source. In the positive ion mode, the capillary voltage was set at $5500 \mathrm{~V}$ and the cone voltage at $80 \mathrm{~V}$. For experiments performed in the negative ion mode, the capillary voltage was $-4500 \mathrm{~V}$ and the cone voltage was $-70 \mathrm{~V}$. In this hybrid instrument, ions were measured using an orthogonal acceleration time-of-flight (oa-TOF) mass analyzer. A quadrupole was used for selection of precursor ions to be further submitted to collision induced dissociation (CID) in MS/MS experiments. In MS, accurate mass measurements were performed using two reference ions from a poly(ethylene glycol) internal standard, according to a procedure described elsewhere [33]. The precursor ion was used as the reference for accurate measurements of product ion $\mathrm{m} / \mathrm{z}$ ratio in MS/MS spectra. $\mathrm{MS}^{3}$ experiments were performed with a 3200 Q-TRAP mass spectrometer (Applied Biosystems SCIEX) equipped with an electrospray ionization source operated in positive mode. The capillary voltage was set at $5500 \mathrm{~V}$ and the cone voltage at $80 \mathrm{~V}$. Primary precursor ions generated in the ion source were selected in the quadrupole analyzer and submitted to CID in a collision cell. Secondary precursor ions produced during collisions were selected and then fragmented in a linear ion trap. In both instruments, air was used as the nebulizing gas (10 psi) whereas nitrogen was used as the curtain gas (20 psi) as well as the collision gas. Collision energy was set according to the experiments. Instrument control, data acquisition and data processing of all experiments were achieved using Analyst software (QS 2.0 and 1.4.1 for the QqTOF and the QqTrap instruments, respectively) provided by Applied Biosystems. Sample solutions were introduced in the ionization source at a 5 $\mu \mathrm{L} . \mathrm{min}^{-1}$ flow rate using a syringe pump.

\section{Nucleophile Addition}

The nitroxide adduct 1a was produced in benzene by mixing MNP (40 $\mathrm{mM})$ and GSH $(5 \mathrm{mM})$ for $20 \mathrm{~min}$ in dark before adding $\mathrm{H}_{2} \mathrm{O}_{2}(0.3 \% \mathrm{vol} / \mathrm{vol})$. The nitroxide adduct 2a was produced in benzene by mixing $\mathrm{H}_{2} \mathrm{O}_{2}(0.3 \% \mathrm{vol} / \mathrm{vol})$, MNP (40 mmol) and GSH (5 $\mathrm{mM}$ ). The system was then irradiated for $30 \mathrm{~s}$ using visible light. For each experiment, $300 \mu \mathrm{L}$ of the reaction medium was prepared and an aliquot (ca. 30 $\mu \mathrm{L}$ ) of the organic phase was analyzed by EPR spectroscopy. MS samples were prepared by diluting the reaction medium $(1 / 10)$ in a methanolic solution of ammonium acetate $(3 \mathrm{mM})$. 


\section{Results and Discussion}

\section{Electron Paramagnetic Resonance Analysis}

Depending on the experimental conditions, the reaction between 2-methyl 2-nitrosopropane (MNP) and reduced glutathione (GSH) in oxidative medium could generate two different radical species characterized by the EPR signals shown in Figure S1 in Supplementary Material, which can be found in the electronic version of this article.

The first paramagnetic compound 1a is formed by mixing MNP and GSH for 20 min before adding $\mathrm{H}_{2} \mathrm{O}_{2}$ in dark condition. The triplet EPR signal is characteristic of a nitroxide $\left(\mathrm{a}_{\mathrm{N}}=18.2 \mathrm{G}, \mathrm{g}=2.0067\right)$, the value of the hyperfine coupling constant with nitrogen nucleus, $\mathrm{a}_{\mathrm{N}}$, indicating the presence of a sulfur atom directly bound to the nitroxide nitrogen. Similar EPR parameters have already been reported for spin adducts obtained after trapping thiyl radicals by MNP [23-25]. It should be noted that this signal was not observed before addition of $\mathrm{H}_{2} \mathrm{O}_{2}$ whereas another three line EPR spectrum was detected (with $\mathrm{a}_{\mathrm{N}}=15.5 \mathrm{G}$ and $\mathrm{g}=2.0059$ ) that could be assigned to di-tert-butyl nitroxide (DTBN), a wellknown MNP thermo- or photo-degradation product (Scheme 1). This DTBN EPR signal becomes negligible as the concentration of $\mathbf{1 a}$ increases. Based on EPR data, a structure could be proposed for the nitroxide 1a, as shown in Scheme $\mathbf{1 .}$

The second experimental set up consists of irradiating the sample containing all chemicals (MNP, GSH, and $\mathrm{H}_{2} \mathrm{O}_{2}$ ) with visible light. An EPR signal consisting of a large triplet due to a unique hyperfine coupling of the unpaired electron with the nitrogen nucleus $\left(\mathrm{a}_{\mathrm{N}}=\right.$ 27.2 $\mathrm{G}, \mathrm{g}=2.0058$ ) was thus observed in addition to the previously described DTBN degradation product, as shown in Figure S1. Such high $a_{N}$ values (27.2 G) indicate a direct addition of a function with a strong mesomere electrodonor effect on the nitroso nitrogen. In particular, oxy-nitroxides generally exhibit EPR spectra with an $a_{N}$ value in the 25-30 G range [34-37]. Note also that these EPR parameters are not consistent with a nitroxide bearing a sulfoxide group, since such a $\mathrm{RS}(\mathrm{O})$ $\mathrm{N}\left(\mathrm{O}^{\bullet}\right)-\mathrm{R}$ compound would exhibit an $\mathrm{a}_{\mathrm{N}}$ value in the 12-13 $G$ range [38]. From these results, this second signal could be attributed to the radical species $\mathbf{2 a}$, which structure is shown in Scheme 1. However, it is worth mentioning that the $N$-tert-butoxy- $N$-tert-butylnitroxide 3, which shows almost identical EPR parameters in benzene $\left(a_{N}=26.6 \mathrm{G}, \mathrm{g}=2.0058\right)$ would be formed in the presence of $\mathrm{H}_{2} \mathrm{O}_{2}$ in benzene as a photo-degradation byproduct of MNP. To check whether the nitroxide 2a was actually formed under our experimental conditions, we also recorded with a lower modulation amplitude $(0.5 \mathrm{G})$ the EPR signal obtained after irradiating for $30 \mathrm{~s}$ with visible white light a benzene solution containing $\mathrm{MNP}(40 \mathrm{mM})$ and $\mathrm{H}_{2} \mathrm{O}_{2}(0.3 \%)$ in the absence or in the presence of GSH $(5 \mathrm{mM})$. In the absence of GSH, the EPR signal observed consisted of two triplets, corresponding to DTBN $\left(\mathrm{a}_{\mathrm{N}}=15.4 \mathrm{G}, \mathrm{g}=\right.$ $2.0059)$ and to $3\left(\mathrm{a}_{\mathrm{N}}=27.0 \mathrm{G}, \mathrm{g}=2.0058\right)$. The EPR spectra obtained in the presence of GSH showed broad lines, which could suggest that both the alkoxynitroxide $\mathbf{3}$ and the sulfoxy-nitroxide $\mathbf{2 a}$ would contribute to the signal, though the spectral resolution was not high enough to allow a clear distinction between these two species.

EPR detection analysis allows to make some assumptions about the detected species. In particular, the thio-nitroxide 1a, called S-adduct in the following, could be distinguished from the $O$-adduct 2 a or from the alkoxynitroxide 3 . However, the structure of observed radicals could not be more precisely defined by this technique, which focuses on the detection of the sole paramagnetic function. Moreover, it should also be noted that EPR parameters of a given spin adduct may vary significantly with the nitroxide environment, for instance with the solvent polarity. Last, the presence of 2a in the medium was not clearly established on the basis of EPR analysis. To gain detailed structural characteristics, MS and MS/MS experiments were performed on both reaction products.
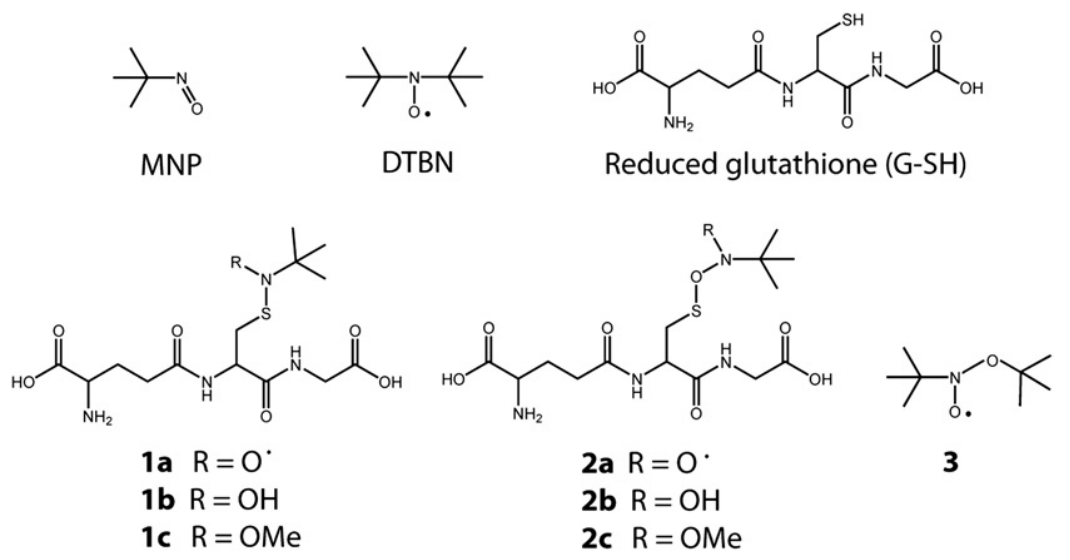

3

Scheme 1. Chemical structure of reagents and proposed structure for the glutathione conjugates produced after reactions between glutathione and MNP. 


\section{Structural Characterization by Mass Spectrometry}

The reaction medium in which the $S$-adduct 1 a was produced was first submitted to electrospray ionization (ESI) after diluting the organic solution in methanol containing $3 \mathrm{mM}$ ammonium acetate. Although detected by EPR, the nitroxide 1a could not be observed as a protonated molecule, expected at $m / z$ 394.2. It should be noted that the use of nonprotic solvents did not allow the expected nitroxide to be detected as an ionized species. In the following, methanol was thus selected as the preferential solvent for dilution before mass analysis since it was shown to promote a better ionization of other molecules of interest. In particular, the positive mode ESI-MS spectrum shows a peak at $\mathrm{m} / \mathrm{z}$ $395.1584\left(\mathrm{C}_{14} \mathrm{H}_{27} \mathrm{~N}_{4} \mathrm{O}_{7} \mathrm{~S}^{+}\right.$, error: $\left.-2.8 \mathrm{ppm}\right)$, which could be assigned based on accurate mass measurement, to the protonated hydroxylamine $\left[\mathbf{1 b}+\mathrm{H}^{+}(\right.$Scheme $\mathbf{1})$. Another cation, detected at $\mathrm{m} / \mathrm{z} 409.1736\left(\mathrm{C}_{15} \mathrm{H}_{29} \mathrm{~N}_{4} \mathrm{O}_{7} \mathrm{~S}^{+}\right.$, error: $-3.8 \mathrm{ppm}$ ), would result from the protonation of the O-methyl hydroxylamine 1c (Scheme 1). The same species could also be ionized in the negative mode and were detected as deprotonated molecules, $[\mathbf{1 b}-\mathrm{H}]^{-}$and $[\mathbf{1 c}-$ $\mathrm{H}^{-}$, at $\mathrm{m} / \mathrm{z} 393.1$ and 407.2, respectively. Tandem mass spectrometry experiments were then performed to validate these structural assignments.

At low collision energy, CID of $[\mathbf{1 b}+\mathbf{H}]^{+}$at $m / z$ 395.2 gave rise to the formation of a main product ion at $\mathrm{m} / \mathrm{z} 322.1$ as shown in Figure 1a. Accurate mass measurement $\left(\mathrm{m} / \mathrm{z}\right.$ 322.0703, $\mathrm{C}_{10} \mathrm{H}_{16} \mathrm{~N}_{3} \mathrm{O}_{7} \mathrm{~S}^{+}$, error: -0.1 $\mathrm{ppm}$ ) indicates this ion was formed after the elimination of a $\mathrm{C}_{4} \mathrm{H}_{11} \mathrm{~N}$ neutral from the precursor ion. A combined loss of a 2-methyl-1-propene molecule, from the tert-butyl group, and ammonia would account for the formation of this $\mathrm{m} / \mathrm{z} 322.1$ product ion. Increasing the collision energy leads to the production of several additional product ions, most of which could be accounted for by considering dissociation of the $\mathrm{m} / \mathrm{z}$ 322.1 product ion, as supported by $\mathrm{MS}^{3}$ experiments and accurate mass data. In particular, dehydration of $\mathrm{m} / \mathrm{z} 322.1$ was observed to occur from a $30 \mathrm{eV}$ collision energy (laboratory frame). The MS/MS behavior of the protonated hydroxylamine $\mathbf{1 b}$ greatly differs from fragmentation patterns described for several glutathione conjugates, characterized in positive ion mode tandem mass spectrometry by the loss of the $129 \mathrm{Da}$ pyroglutamic acid neutral [13-17]. This suggests that a nitroso function on the thiol moiety would completely modify dissociation of the ion in the gas-phase. However, dissociation reactions observed for $[\mathbf{1} b+\mathrm{H}]^{+}$remain poorly informative. In contrast, data obtained while submitting the deprotonated $\mathbf{1 b}$ molecule, $[\mathbf{1 b}-\mathrm{H}]^{-}$, to CID (Figure 1b) allows the structure proposed for this hydroxylamine to be validated, based on fragmentation rules recently established for glutathione conjugates ionized in the negative mode [19]. Since the dissociation pathways of such deprotonated molecules have already been thoroughly discussed by Dieckhaus et al. [19], they will only be briefly described here. The intense $\mathrm{m} / \mathrm{z} 272.1$
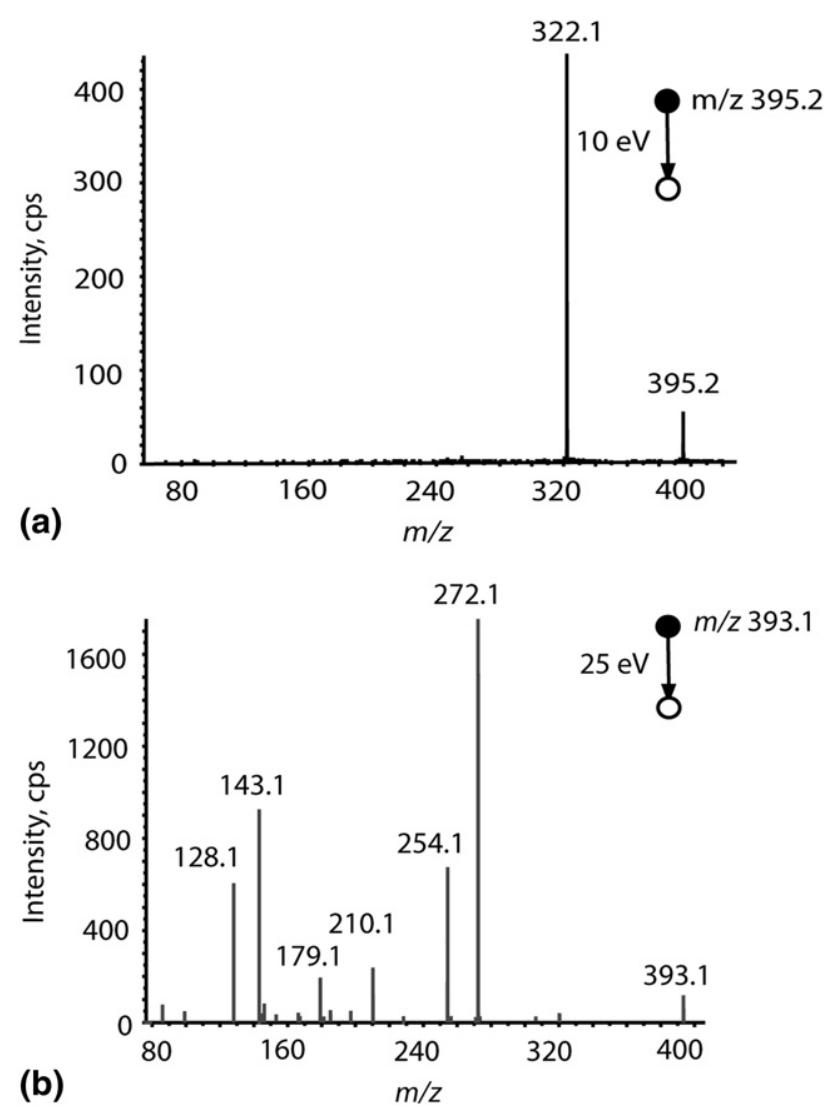

Figure 1. ESI-MS/MS spectrum of $(\mathbf{a})[\mathbf{1} \mathbf{b}+\mathbf{H}]^{+}$at $\mathrm{m} / \mathrm{z} 395.2$ using a $10 \mathrm{eV}$ collision energy and $(\mathbf{b})[\mathbf{1 b}-\mathbf{H}]^{-}$at $\mathrm{m} / \mathrm{z} 393.1 \mathrm{using}$ a $25 \mathrm{eV}$ collision energy (laboratory frame).

product ion in Figure $1 \mathrm{~b}$ would arise from the elimination of a $N$-hydroxy- $N$-mercapto-tert-butylamine neutral from the $m / z 393.1$ precursor ion. This result implies that the reaction between MNP and glutathione has produced a species which contains a covalent S-N bond. The soobtained deprotonated $\gamma$-glutamyl-dehydroalanyl-glycine further dissociates to generate product ions at $\mathrm{m} / \mathrm{z} 254.1$, 210.1, 179.1, 143.1 and 128.1. The cleavage of the $\gamma$-GluCys amide bond in $\mathrm{m} / \mathrm{z} 272.1$ would produce $\mathrm{m} / \mathrm{z} 143.1$ or $m / z$ 128.1, depending on which carboxylic acid function is deprotonated. Dehydration of $m / z 272.1$ would produce $\mathrm{m} / \mathrm{z} 254.1$, which would further decarboxylate to yield $\mathrm{m} / \mathrm{z} 210.1$ or eliminate a glycine residue via hydrogen rearrangement to produce $m / z$ 179.1. As previously reported by Dieckhaus et al. [19], it is shown here that neither the cation nor the anion obtained after electrospray of the same species follows some common trends.

Following the same approach as for $\mathbf{1} \mathbf{b}$, the $\mathrm{m} / \mathrm{z} 409.2$ ion assigned to the protonated methoxyamine $\left(\mathrm{CH}_{3}\right)_{3} \mathrm{C}-$ $\mathrm{N}\left(\mathrm{OCH}_{3}\right)$-S-G (called O-methyl hydroxylamine in the following) $[1 \mathrm{c}+\mathrm{H}]^{+}$was submitted to CID and the so-obtained MS/MS spectrum is presented in Figure 2. It should first be noted that, in contrast to the hydroxylamine $\mathbf{1 b}$, dissociation of protonated $\mathbf{1 c}$ did not proceed via the elimination of a 73 Da neutral. First, a 


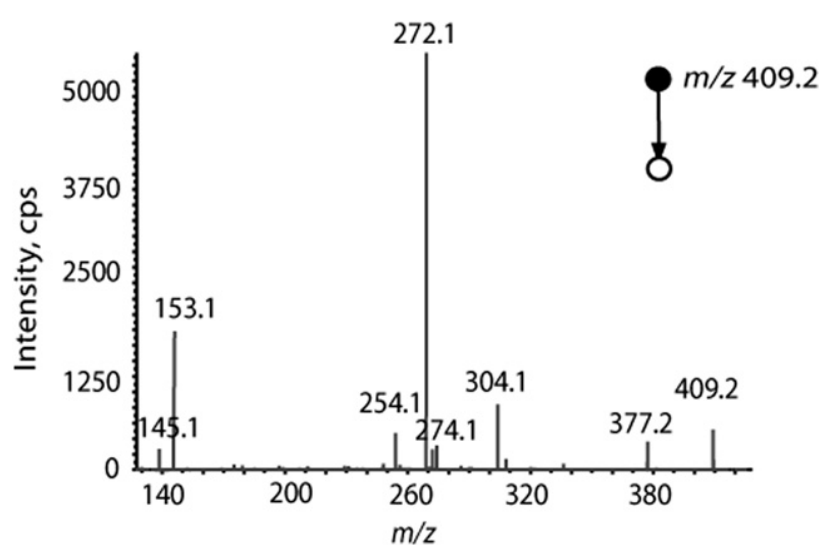

Figure 2. ESI-MS/MS spectrum of $[1 \mathrm{c}+\mathrm{H}]^{+}$at $m / z 409.2$ using a $15 \mathrm{eV}$ collision energy (laboratory frame).

methanol molecule would be released after protonation of the methoxy function to give rise to the product ion at $m / z 377.1514\left(\mathrm{C}_{14} \mathrm{H}_{25} \mathrm{~N}_{4} \mathrm{O}_{6} \mathrm{~S}^{+}\right.$, error: $\left.+6.5 \mathrm{ppm}\right)$. The $73 \mathrm{Da}$ loss, corresponding to the elimination of a $\mathrm{C}_{4} \mathrm{H}_{11} \mathrm{~N}$ neutral, would then occur from this $\mathrm{m} / \mathrm{z} 377.2$ to generate $\mathrm{m} / \mathrm{z} 304.0599\left(\mathrm{C}_{10} \mathrm{H}_{14} \mathrm{~N}_{3} \mathrm{O}_{6} \mathrm{~S}^{+}\right.$, error: +0.4 ppm). Alternatively, dissociation of $\mathrm{m} / \mathrm{z} 377.2$ could produce a tert-butylaminethiol neutral and the high abundance product ion at $m / z 272.0862\left(\mathrm{C}_{10} \mathrm{H}_{14} \mathrm{~N}_{3} \mathrm{O}_{6}{ }^{+}\right.$, error: $-5.5 \mathrm{ppm})$. Further dissociation of this $\mathrm{m} / z 272.1$ ions would give rise to $\mathrm{m} / \mathrm{z} 254.0776\left(\mathrm{C}_{10} \mathrm{H}_{12} \mathrm{~N}_{3} \mathrm{O}_{5}{ }^{+}\right.$, error: $+2.1 \mathrm{ppm}$ ) after elimination of a water molecule and to $\mathrm{m} / \mathrm{z} 153.0671\left(\mathrm{C}_{7} \mathrm{H}_{9} \mathrm{~N}_{2} \mathrm{O}_{2}{ }^{+}\right.$, error: $\left.+8.1 \mathrm{ppm}\right)$ after the release of $\mathrm{H}(\mathrm{CO}) \mathrm{NHCH}(\mathrm{OH}) \mathrm{COOH}$. Finally, the low abundance product ion detected at $\mathrm{m} / \mathrm{z} 145.0615$ $\left(\mathrm{C}_{5} \mathrm{H}_{9} \mathrm{~N}_{2} \mathrm{O}_{3}{ }^{+}\right.$, error: $\left.+5.0 \mathrm{ppm}\right)$ would correspond to the dissociation of the precursor ion at $\mathrm{m} / \mathrm{z} 409.2$, consisting of the cyclization of the glutamic acid part of the glutathione conjugate. As previously indicated in the case of $\mathbf{1 b}$, more straightforward structural insights could be obtained for $1 \mathrm{c}$ in negative mode analysis. Indeed, all product ions typically expected to be formed during CID of deprotonated glutathione-based thiol adduct [19] were observed in the MS/MS spectrum of $[1 \mathrm{c}-\mathrm{H}]^{-}$(i.e., $\mathrm{m} / \mathrm{z} 272,254,210,179,143$, and 128). In addition, an intense peak observed at $\mathrm{m} / \mathrm{z} 304.1$, as well as a lower abundance $\mathrm{m} / \mathrm{z} 377.2$ ion, would have, respectively, been formed after the loss of tert-butyl methoxylamine and methanone from the $\mathrm{m} / \mathrm{z} 407.2$ precursor ion.

The nitroxide 1a could be strongly stabilized via homolytic association with a methyl radical, which could be produced in the medium due to the decomposition of the methoxynitroxide obtained after addition of methanol onto MNP. Alternatively, the same O-methyl hydroxylamine 1c could have been formed via a nucleophile substitution either on the hydroxylamine nitrogen, methanol behaving here as the nucleophile [39], or on the carbon of the protonated methanol, attacked by the hydroxylamine oxygen atom. Production of $1 \mathrm{c}$ has probably occurred in the liquid phase during dilution of the reaction medium with methanol rather than during the ESI process. Indeed, 1a is no longer detected in EPR when methanol is used as the solvent of the reaction medium. Hence, formation of such O-methyl hydroxylamine appears as an efficient and simple derivatization reaction, which allows the stabilization of hardly detectable radical species such as $\mathbf{1 a}$ and thus their complete MS/MS structural characterization.

Structural characterization of the $O$-adduct 2a (Scheme 1) was of particular interest since such a species has never been reported, neither by spin trapping nor by nucleophile reaction. Positive ion mode ESI-MS analysis of a methanolic solution of the reaction medium did not show, as in the case of $\mathbf{1 a}$, the signal expected at $\mathrm{m} / \mathrm{z} 410.2$ for $[2 \mathrm{a}+\mathrm{H}]^{+}$. In contrast, a high abundance peak was observed at $m / z 411.1577\left(\mathrm{C}_{14} \mathrm{H}_{27} \mathrm{~N}_{4} \mathrm{O}_{8} \mathrm{~S}^{+}\right.$, error: $\left.+8 \mathrm{ppm}\right)$ and assigned to the protonated hydroxylamine $\mathbf{2 b}$ (Scheme 1). A signal was also detected in the negative ion mode for $[\mathbf{2} \mathbf{b}-\mathbf{H}]^{-}$at $m / z$ 409.1. CID of $[\mathbf{2} \mathbf{b}+\mathbf{H}]^{+}$gives rise to several product ions (Figure $3 a$ ) for which dissociation pathways could be proposed (Figure S2). In particular, the loss of a 129 Da neutral expected to occur from protonated glutathione conjugates was observed to yield $\mathrm{m} / \mathrm{z}$ 282.1. Moreover, the peak at $\mathrm{m} / \mathrm{z} 306.1$ could be
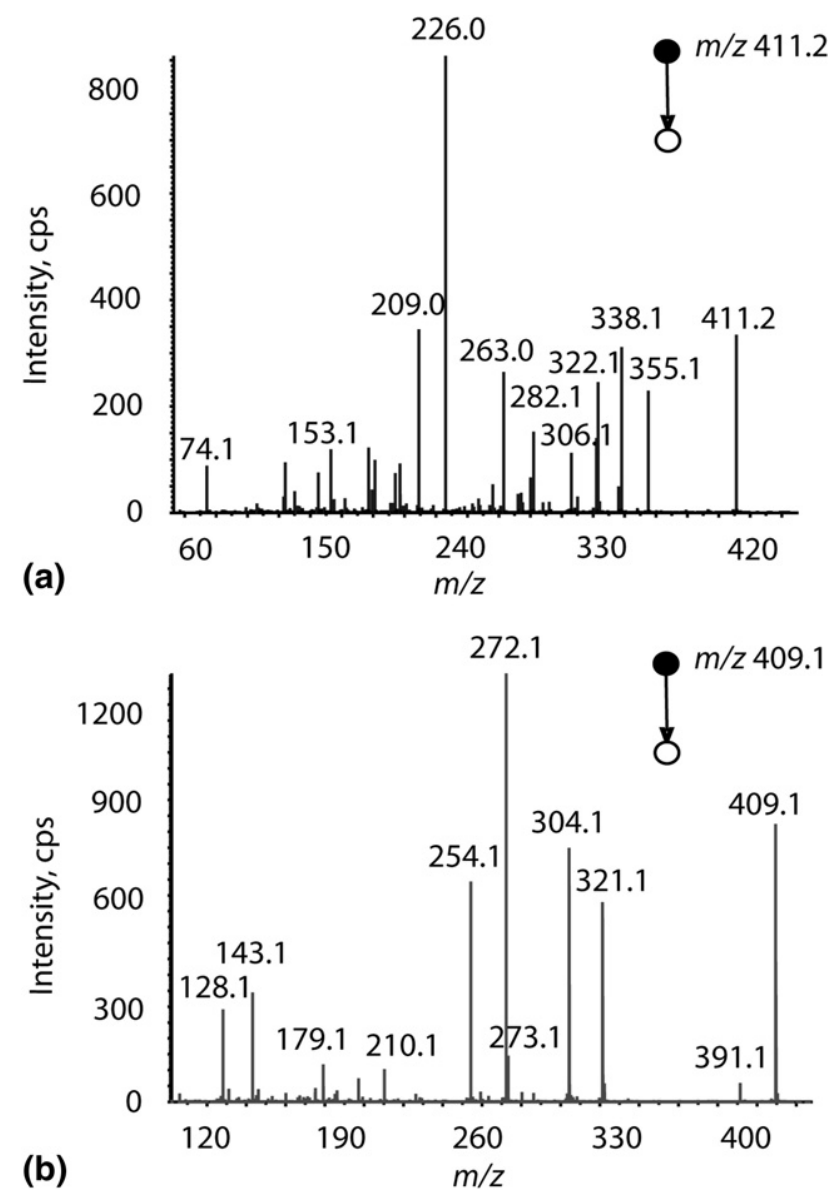

Figure 3. ESI-MS/MS spectrum of (a) $[2 \mathbf{b}+\mathrm{H}]^{+}$at $m / z 411.2$ and (b) $[\mathbf{2 b}-\mathrm{H}]^{-}$at $\mathrm{m} / \mathrm{z} 409.1$, using a $20 \mathrm{eV}$ collision energy (laboratory frame). 
assigned to a protonated molecule in which the thiol function in glutathione is oxidized, arising from the release of $N, N$-dihydroxy-2-methylpropan-2-amine from the precursor ion. Finally, a tert-butylhydroxylamine neutral loss from $m / z 411.2$ would produce $m / z$ 322.1. Again, results obtained in negative ion mode more clearly support the structural assumption proposed for $2 \mathbf{a}$ based on EPR data. First, the MS/MS spectrum of $[2 \mathbf{b}-\mathrm{H}]^{-}$ displays all product ions expected for such a deprotonated glutathione conjugates (Figure 3b). More interestingly, two main additional peaks are also observed at $\mathrm{m} / \mathrm{z} 321.1$ and $m / z$ 304.1. As illustrated in Scheme 2, the $m / z 304.1$ product ion would correspond to a deprotonated molecule in which the thiol function in glutathione is oxidized, as a result of the release of a $N$-dihydroxy-2-methylpropan2-amine molecule from the $m / z 409.1$ precursor ion. The nature of this eliminated neutral further validates the existence of the N-OS bond in the glutathione conjugate 2b. A homolytic bond cleavage, which induces the loss of a hydroxyl-tert-butylamino radical, has to be envisaged to account for the formation of the $m / z 321.1$ product ion, in which delocalization of the unpaired electron from the oxygen to the sulfur atom would enhance this ion stability, as indicated in Scheme 2. This $m / z 321.1$ product ion further dissociates to a small extent via the release of a SO neutral to yield the low abundance $m / z 273.1$ ion. Interestingly, no MS signal related to a $2 c$ species (that is, $m / z 425.2$ in the positive mode or $\mathrm{m} / \mathrm{z} 423.2$ in the negative mode) could be observed in the ESI-MS spectrum obtained from this sample. The absence of this $O$-methyl hydroxylamine homologue of $\mathbf{2} \mathbf{b}$ could be explained, in the case of a radical process, by a higher mesomere donor effect due to an $\mathrm{O}-\mathrm{N}$ bond in $\alpha$ position toward the nitroxide function in 2a. Indeed, the limiting mesomeric form with the unpaired electron on the nitrogen atom would be favored over the one with the unpaired electron on the oxygen atom, as indicated by the high hyperfine coupling constant value $\left(\mathrm{a}_{\mathrm{N}}=27.2 \mathrm{G}\right)$ measured in EPR. It could thus be concluded that, in contrast to $1 \mathbf{a}$, the nitroxide $\mathbf{2 a}$ would be much less reactive toward carbon-centered radicals.

Although nitroxide species could not be directly observed in mass spectrometry, detailed information obtained from MS/MS analysis of related compounds in both reaction media allows to validate structural assumptions proposed for $\mathbf{1 a}$ and $\mathbf{2 a}$ based on EPR results. The mechanism of reactions involved between glutathione and MNP could thus be further studied.

\section{Considerations on the Reaction Mechanisms}

The formation of both nitroxides $1 \mathbf{a}$ and $\mathbf{2 a}$ could either result from an inverted spin trapping reaction or a Forrester-Hepburn reaction. The inverted spin trapping process [40-42] first consists of the oxidation of the nitroso compound to yield a radical cation, followed by the addition of a nucleophile to form the final nitroxide product. In contrast, the Forrester-Hepburn reaction [43-46] starts with a nucleophile addition, generating a hydroxylamine intermediate, which is further oxidized into nitroxide. These two reactions could sometimes be distinguished based on the species produced after slight modifications of experimental conditions when allowing reduced glutathione to react with MNP.

Indeed, when experiments were performed without the use of $\mathrm{H}_{2} \mathrm{O}_{2}$ as an oxidant, 1a could not be detected in EPR while $\mathbf{1 b}$ was observed in MS. Therefore, $\mathbf{1 b}$ is not only a reduction product of $\mathbf{1 a}$ but also the intermediate hydroxylamine leading to 1a (Scheme 3 ). This result clearly shows that the reaction between the peptide thiol and MNP occurs via a Forrester-Hepburn reaction implying the formation of a hydroxylamine intermediate further oxidized into nitroxide.

In contrast, the need for photoactivation of the medium to generate $\mathbf{2 a}$ could suggest an inverted spin trapping reaction where the spin trap is first excited and further oxidized to produce a radical cation intermediate. Many examples have shown photolysis of nitrones in the presence of an oxidative agent produced a nitrone radical cation which could further react with nucleophiles [40, 41]. Here, the thiol moiety in reduced glutathione would be oxidized to yield a sulfenic acid function, which further adds onto the nitrogen of the MNP (Scheme 3). Alternatively, the sulfenic acid could be generated after a photo-oxidation of $\mathrm{GSH}$ by $\mathrm{H}_{2} \mathrm{O}_{2}$
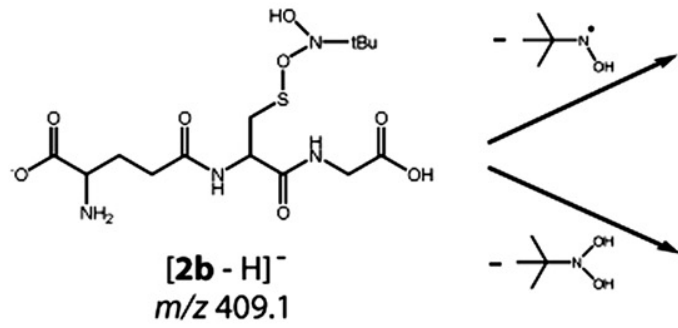

$m / z 409$.
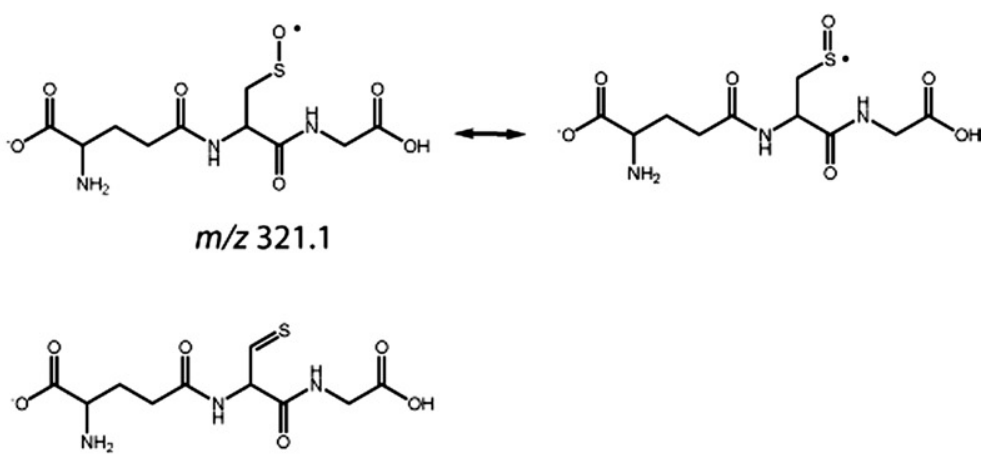

$m / z 304.1$

Scheme 2. Proposed dissociation pathways of $[2 \mathbf{b}-\mathrm{H}]^{-}$at $m / z 409.1$ to account for the formation of $\mathrm{m} / \mathrm{z} 321.1$ and $\mathrm{m} / \mathrm{z} 304.1$ product ions. 


\section{Forres ter Hepburn reaction}

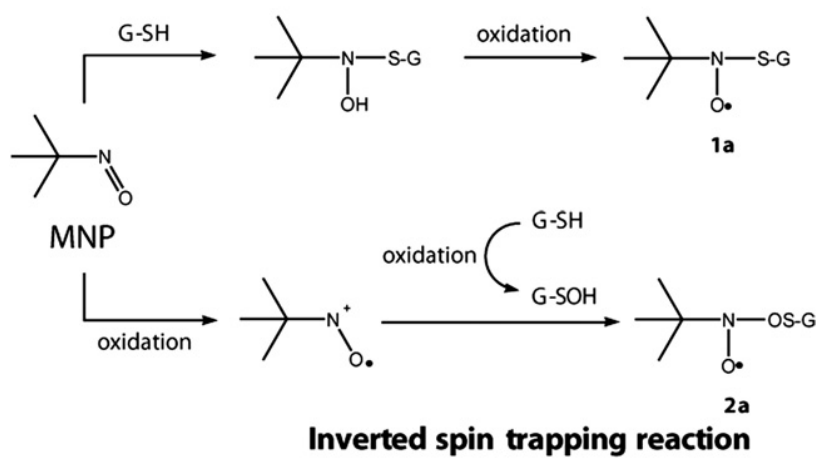

Scheme 3. Different steps of the Forrester Hepburn reaction (yielding 1a) and the inverted spin trapping reaction (giving rise to 2a) between glutathione (GSH) and MNP.

before reacting with MNP according to a ForresterHepburn process. To dismiss one of the two hypotheses, a benzene solution containing GSH $(5 \mathrm{mM})$ and $\mathrm{H}_{2} \mathrm{O}_{2}(0.3 \%)$ was irradiated with visible light for $5 \mathrm{~min}$. Then, MNP $(40 \mathrm{mM})$ was added and the medium was allowed to react for $20 \mathrm{~min}$ in darkness before being submitted to both EPR and MS analysis. An EPR analysis of the medium did not reveal the presence of any paramagnetic species, even after increasing the $\mathrm{H}_{2} \mathrm{O}_{2}$ concentration to $1.2 \%$. In addition, neither the nitroxide $\mathbf{2 a}$ nor any of the diamagnetic species $\mathbf{2} \mathbf{b}$ and $2 \mathrm{c}$ was detected by ESI-MS. These results suggest that a photo-activation of MNP intervenes in the process and invalidate a Forrester-Hepburn mechanism.

\section{Conclusion}

The use of electron paramagnetic resonance in conjunction with direct introduction electrospray-tandem mass spectrometry was shown here to be a powerful tool in the study of the reactions occurring between reduced glutathione and 2-methyl 2-nitrosopropane. Structural assumptions allowed by analysis of EPR signals obtained for the so-formed nitroxides could be validated based on accurate mass measurements and fragmentation pathways of ions produced from the corresponding hydroxylamines, in particular the deprotonated molecules generated in the negative ion mode. The reactivity of reduced glutathione as a nucleophile toward the studied nitroso compound was shown to highly depend on experimental conditions, such as the presence of an oxidant or the irradiation of the reaction medium with visible light. Such an analytical approach would be particularly valuable in biological studies devoted to oxidative stress and antioxidants.

\section{Acknowledgments}

L.C. acknowledges support from Spectropole, the Analytical Facility of Aix-Marseille University, by allowing a special access to the instruments purchased with European Funding (FEDER OBJ21423341).

\section{Appendix A Supplementary Material}

Supplementary material associated with this article may be found in the online version at doi:10.1016/ j.jasms.2009.07.018.

\section{References}

1. Sies, H. Glutathione and Its Role in Cellular Functions. Free Radical. Biol. Med. 1999, 27, 916-921.

2. Mantle, T. J.; Pickett, C. B.; Hayes, J. D. Glutathione S-Transferases and Carcinogenesis; Taylor and Francis: London, 1987.

3. Hayes, J. D.; Pickett, C. B.; Mantle, T. J. Glutathione S-Transferases and Drug Resistance; Taylor and Francis: London, 1990.

4. Larsson, A.; Orrenius, S.; Holmgren, A.; Mannervik, B. Functions of Glutathione. Biochemical, Physiological, Toxicological, and Clinical Aspects; Raven: New York, 1983; pp 99-108.

5. Dolphin, D.; Poulson, R.; Avramovic, D. Glutathione: Chemical, Biochemical, and Medical Aspects; Wiley: New York, 1989.

6. Thatcher, N. J.; Murray, S. Analysis of the Glutathione Conjugate of Paracetamol in Human Liver Microsomal Fraction by Liquid Chromatography Mass Spectrometry. Biomed. Chromatogr. 2001, 15, 374-378.

7. Stockigt, D.; Haebel, S. Identification of Collision-Induced Dissociation Fragments from a Protonated Glutathione Conjugate by Isotope-Specific MS3 Experiments in an Ion Trap. Rapid Commun. Mass Spectrom. 1998, 12, 273-275.

8. Erve, J. C. L.; Deinzer, M. L.; Reed, D. J. Alkylation of Oxytocin by S-(2-Chloroethyl)Glutathione and Characterization of Adducts by Tandem Mass-Spectrometry and Edman Degradation. Chem. Res. Toxicol. $1995,8,414-421$.

9. Ma, S. G.; Subramanian, R. Detecting and Characterizing Reactive Metabolites by Liquid Chromatography/Tandem Mass Spectrometry. J. Mass Spectrom. 2006, 41, 1121-1139.

10. Haroldsen, P. E.; Reilly, M. H.; Hughes, H.; Gaskell, S. J.; Porter, C. J. Characterization of Glutathione Conjugates by Fast Atom Bombardment Tandem Mass-Spectrometry. Biomed. Environ. Mass Spectrom. 1988, 15, 615-621.

11. Murphy, C. M.; Fenselau, C.; Gutierrez, P. L. Fragmentation Characteristic of Glutathione Conjugates Activated by High-Energy Collisions. J. Am. Soc. Mass Spectrom. 1992, 3, 815-822.

12. Levsen, K.; Schiebel, H. M.; Behnke, B.; Dotzer, R.; Dreher, W.; Elend, M.; Thiele, H. Structure Elucidation of Phase II Metabolites by Tandem Mass Spectrometry: An Overview. J. Chromatogr. A 2005, 1067, 55-72.

13. Soglia, J. R.; Harriman, S. P.; Zhao, S.; Barberia, J.; Cole, M. J.; Boyd, J. G.; Contillo, L. G. The Development of a Higher Throughput Reactive Intermediate Screening Assay Incorporating Micro-Bore Liquid ChromatographyMicro-Electrospray Ionization-Tandem Mass Spectrometry and Glutathione Ethyl Ester as an In Vitro Conjugating Agent. J. Pharm. Biomed. Anal. 2004, $36,105-116$.

14. Chen, W. C. G.; Zhang, C. H.; Avery, M. J.; Fouda, H. G. Reactive Metabolite Screen for Reducing Candidate Attrition in Drug Discovery. Adv. Exp. Med. Biol. 2001, 500, 521-524.

15. Castro-Perez, J.; Plumb, R.; Liang, L.; Yang, E. A High-Throughput Liquid Chromatography/Tandem Mass Spectrometry Method for Screening Glutathione Conjugates Using Exact Mass Neutral Loss Acquisition. Rapid Commun. Mass Spectrom. 2005, 19, 798-804.

16. Davoine, C.; Douki, T.; Iacazio, G.; Montillet, J. L.; Triantaphylides, C. Conjugation of Keto Fatty Acids to Glutathione in Plant Tissues. Characterization and Quantification by HPLC-Tandem Mass Spectrometry. Anal. Chem. 2005, 77, 7366-7372.

17. Zhu, M. S.; Ma, L.; Zhang, H. Y.; Humphreys, W. G. Detection and Structural Characterization of Glutathione-Trapped Reactive Metabolites Using Liquid Chromatography-High-Resolution Mass Spectrometry and Mass Defect Filtering. Anal. Chem. 2007, 79, 8333-8341.

18. Baillie, T. A.; Davis, M. R. Mass-Spectrometry in the Analysis of Glutathione Conjugates. Biol. Mass Spectrom. 1993, 22, 319-325.

19. Dieckhaus, C. M.; Fernandez-Metzler, C. L.; King, R.; Krolikowski, P. H.; Baillie, T. A. Negative Ion Tandem Mass Spectrometry for the Detection of Glutathione Conjugates. Chem. Res. Toxicol. 2005, 18, 630-638.

20. Wardman, P.; Vonsonntag, C. Kinetic Factors that Control the Fate of Thiyl Radicals in Cells. Methods Enzymol. 1995, 251, 31-45.

21. Eling, T. E.; Curtis, J. F.; Harman, L. S.; Mason, R. P. Oxidation of Glutathione to Its Thiyl Free-Radical Metabolite by Prostaglandin-H Synthase- a Potential Endogenous Substrate for the Hydroperoxidase. J. Biol. Chem. 1986, 261, 5023-5028.

22. Harman, L. S.; Carver, D. K.; Schreiber, J.; Mason, R. P. One-Electron and 2-Electron Oxidation of Reduced Glutathione by Peroxidases. J. Biol. Chem. 1986, 261, 1642-1648.

23. Stock, B. H.; Schreiber, J.; Guenat, C.; Mason, R. P.; Bend, J. R.; Eling, T. E. Evidence for a Free-Radical Mechanism of Styrene-Glutathione Conjugate Formation Catalyzed by Prostaglandin-H Synthase and Horseradish-Peroxidase. J. Biol. Chem. 1986, 261, 15915-15922. 
24. Felix, C. C.; Reszka, K.; Sealy, R. C. Free-Radicals from Photo-Reduction of Hematoporphyrin in Aqueous-Solution. Photochem. Photobiol. 1983, 37, 141-147.

25. Jin, J.; Wu, L. M.; Zhang, Z. Y. EPR Study of Radicals Generated from Bioactive S-Nitrosothiols and Related Thiols. Magn. Reson. Chem. 2002, 40, 346-352.

26. Kazanis, S.; McClelland, R. A. Electrophilic Intermediate in the Reaction of Glutathione and Nitrosoarenes. J. Am. Chem. Soc. 1992, 114, 3052-3059.

27. Eyer, P. Reactions of Oxidatively Activated Arylamines with ThiolsReaction-Mechanisms and Biologic Implications-an Overview. Environ. Health Perspect. 1994, 102, 123-132.

28. Naisbitt, D. J.; Oneill, P. M.; Pirmohamed, M.; Park, B. K. Synthesis and Reactions of Nitroso Sulphamethoxazole with Biological Nucleophiles: Implications for Immune Mediated Toxicity. Bioorg. Med. Chem. Lett. 1996, 6, 1511-1516.

29. Gallemann, D.; Greif, A.; Eyer, P.; Wagner, H. U.; Sonnenbichler, J.; Sonnenbichler, I.; Schafer, W.; Buhrow, I. Additional Pathways of S-Conjugate Formation During Interaction of 4-Nitrosophenetole with Glutathione. Chem. Res. Toxicol. 1998, 11, 1411-1422.

30. Tuccio, B.; Lauricella, R.; Charles, L. Characterization of Free Radical Spin Adducts of the Cyclic $\beta$-Phosphorylated Nitrone DEPMPO Using Tandem Mass Spectrometry. Int. J. Mass Spectrom. 2006, 252, 47-53.

31. Hassan, I. E.; Charles, L.; Lauricella, R.; Tuccio, B. Detection and Identification of Various Carbon-Centered Free Radicals Using NArylketonitrones: A Spin Trapping/EPR/MS Study. New J. Chem. 2008, 32, 680-688

32. Duling, D. R. Simulation of Multiple Isotropic Spin-Trap Epr-Spectra. J. Magn. Reson. Ser. B 1994, 104, 105-110.

33. Charles, L. Influence of Internal Standard Charge State on the Accuracy of Mass Measurements in Orthogonal Acceleration Time-of-Flight Mass Spectrometers. Rapid Commun. Mass Spectrom. 2008, 22, 151-155.

34. Sargent, F. P.; Gardy, E. M. Radical Yields in Irradiated Methanol and Ethanol-Electron-Spin Resonance and Spin Trapping Method. J. Phys. Chem. 1976, 80, 854-856.

35. Forshult, S.; Lagercrantz, C.; Torssell, K. Use of Nitroso Compounds as Scavengers for the Study of Short-Lived Free Radicals in Organic Reactions. Acta Chem. Scand. 1969, 23, 522-530.
36. Mao, S. W.; Kevan, L. Electron Paramagnetic Resonance Studies of Spin Trapping of the Primary Neutral Radicals Formed in $\gamma$-Irradiated Methanol. Chem. Phys. Lett. 1974, 24, 505-507.

37. Lucarini, M.; Pedulli, G. F.; Alberti, A.; Benaglia, M. Kinetic EPR Studies of the Decay of Tert-Butyl Alkoxy, Silyloxy, Germyloxy, and Stannyloxy Nitroxides. J. Am. Chem. Soc. 1992, 114, 9603-9607.

38. Jin, J.; Wu, L. M.; Zhang, Z. Y. Electron Paramagnetic Resonance Study of Nitroxides Generated from Nitric Oxide by Reaction with Transient Radicals. Magn. Reson. Chem. 2002, 40, 284-288.

39. Yamamoto, F.; Oae, S. Nucleophilic-Substitution on Trivalent Nitrogen Atom-Menschutkin Type Reaction of O-2,4-Dinitrophenylhydroxylamine with Uncharged Nucleophiles. Bull. Chem. Soc. Jpn. 1975, 48, 77-79.

40. Eberson, L. Inverted Spin Trapping Reactions between the Radical Cation of $\alpha$-Phenyl-N-Tert-Butylnitrone and Ionic and Neutral Nucleophiles. J. Chem. Soc. Perkin Trans. 1992, 2, 1807-1813.

41. Eberson, L. Inverted Spin-Trapping. 3. Further-Studies on the Chemical and Photochemical Oxidation of Spin Traps in the Presence of Nucleophiles. J. Chem. Soc. Perkin Trans. 1994, 2, 171-176.

42. Eberson, L.; Lind, J.; Merenyi, G. Inverted Spin-Trapping. 4. Application to the Formation of Imidyl Spin Adducts from N-Haloimides. J. Chem. Soc., Perkin Trans. 1994, 2, 1181-1188.

43. Alberti, A.; Carloni, P.; Eberson, L.; Greci, L.; Stipa, P. New Insights into N-tert-Butyl- $\alpha$-Phenylnitrone (PBN) as a Spin Trap. 2. The Reactivity of PBN and 5,5-Dimethyl-4,5-Dihydropyrrole N-Oxide (DMPO) toward N-Heteroaromatic Bases. J. Chem. Soc. Perkin Trans. 1997, 2, 887-892.

44. Grigorev, I. A.; Volodarsky, L. B.; Starichenko, V. F.; Kirilyuk, I. A Synthesis of Stable Nitroxides with Amino-Groups and Fluorine-Atoms at Alpha-Carbon of the Radical Center. Tetrahedron. Lett. 1989, 30, $751-754$.

45. Dopp, D.; Greci, L.; Noureldin, A. M. Indolenine Oxides. 8. Reaction of 5,7-Di-tert-Butyl-3,3-Dimethyl-3h-Indole 1-Oxide with Grignard-Reagents-a New Stable Aminyl Oxide (Nitroxide). Chem. Ber. 1983, 116, 2049-2057.

46. Kirilyuk, I. A.; Shevelev, T. G.; Morozov, D. A.; Khromovskih, E. L.; Skuridin, N. G.; Khramtsov, V. V.; Grigor'ev, I. A. Grignard Reagent Addition to 5-Alkylamino-4H-Imidazole 3-Oxides: Synthesis of New pH-Sensitive Spin Probes. Synthesis 2003, 871-878. 